\title{
Drug-related cancers: Analyses of head and neck cases reported in the literature
}

\author{
Hamed Mortazavi ${ }^{1, A, E, F}$, Behrad Rahbani Nobar ${ }^{2, B-D}$, Shervin Shafiei ${ }^{3, E, F}$, Nima Ahmadi ${ }^{2, B-D}$ \\ ${ }^{1}$ Department of Oral Medicine, School of Dentistry, Shahid Beheshti University of Medical Sciences, Tehran, Iran \\ ${ }^{2}$ Dentistry student, School of Dentistry, Shahid Beheshti University of Medical Sciences, Tehran, Iran \\ ${ }^{3}$ Department of Oral and Maxillofacial Surgery, School of Dentistry, Shahid Beheshti University of Medical Sciences, Tehran, Iran \\ A - research concept and design; $B$ - collection and/or assembly of data; $C$ - data analysis and interpretation; \\ $D$ - writing the article; $\mathrm{E}$ - critical revision of the article; $\mathrm{F}$ - final approval of the article
}

Address for correspondence

Shervin Shafiei

E-mail: shervin.shafee@gmail.com

\section{Funding sources}

None declared

Conflict of interest

None declared

Received on September 4, 2020

Reviewed on November 28, 2020

Accepted on December 3, 2020

Published online on June 25, 2021

Cite as

Mortazavi H, Rahbani Nobar B, Shafiei S, Ahmadi N.

Drug-related cancers: Analyses of head and neck cases reported

in the literature. Dent Med Probl. 2021;58(2):267-280.

doi:10.17219/dmp/131116

DOI

$10.17219 / \mathrm{dmp} / 131116$

Copyright

○) 2021 by Wroclaw Medical University

This is an article distributed under the terms of the

Creative Commons Attribution 3.0 Unported License (CC BY 3.0)

(https://creativecommons.org/licenses/by/3.0/).

\begin{abstract}
Background. Recent advances have attributed carcinogenic potential to pharmacotherapy. Cancers of the head and neck region are no exception.

Objectives. This descriptive investigation aimed to identify studies reporting on drugs that have contributed to cancer development in the head and neck region.

Material and methods. Online databases were searched for relevant articles and their data were summarized, including age, gender, main drug classification and name, additional drugs, primary disorders, drug-related cancers, and the site of each drug-related cancer.

Results. The mean age of the patients included in this analysis was 52.9 years. However, drug-related head and neck cancers (DR HNCS) were most prevalent in persons over 60 years of age. Overall, these cancers were more prevalent in females than in males (1.33/1). The HNC-related drugs could mainly be categorized into 3 groups, namely, immunomodulatory/immunosuppressive, chemotherapeutic and chemoprotective drugs, while the most frequently used additional drugs across the studies were corticosteroids. The 5 most prevalent primary conditions for which the patients had received pharmacotherapy were organ transplantations, lymphoproliferative disorders (LPD), rheumatoid arthritis (RA), Epstein-Barr virus (EBV) infection, and bone sarcoma. The most prevalent HNCs were squamous cell carcinoma (SCCS), thyroid cancers (including papillary and follicular thyroid carcinomas), LPD, and mucoepidermoid/acinic cell carcinomas, which occurred mostly in the oral cavity, neck, salivary glands, pharynx/larynx, and head/face.

Conclusions. This study was the first of its kind to analyze and discuss the aforementioned findings regarding the head and neck region in depth. Clinicians should familiarize themselves with DR HNC cases to effectively screen suspected patients.
\end{abstract}

Keywords: drugs, malignancy, pharmacotherapy, carcinogenesis, head and neck cancer 


\section{Introduction}

Carcinogenesis is defined as the transformation of normal cells into cancer cells, and can be attributed to a number of factors, including pharmacotherapy. ${ }^{1-3}$ The therapeutic use of drugs may cause long-term toxicities ${ }^{4}$ or immunosuppression, ${ }^{5,6}$ which can facilitate cancer development. The subsequent malignancies may present as ulceration, ${ }^{7,8}$ hyperplasia ${ }^{9,10}$ or lymphoproliferative disorders. ${ }^{5,11}$ Although drugs are tested in various ways to ensure their safety, they may not be safe for human use if carcinogenesis is taken into account. ${ }^{12}$ This is because of the relatively non-specific nature of the tests which are used for determining drug toxicity. ${ }^{13,14}$ Head and neck cancers (HNC) are no exception to drug-related (DR) cancers of the human body. ${ }^{2,15}$

Head and neck cancers are defined as cancers occurring within the mouth, pharynx, larynx, nose, paranasal sinuses, thyroid, parathyroid, salivary glands, and cervical esophagus; malignancies related to the skin in this region are also counted as HNCs. ${ }^{16}$ Head and neck cancers are the $9^{\text {th }}$ most prevalent malignancies, with over 650,000 cases worldwide each year. ${ }^{17}$ Although risk factors for $\mathrm{HNC}$, such as smoking, ${ }^{18,19}$ alcohol consumption, ${ }^{18,19}$ and human papillomavirus (HPV) ${ }^{18,19}$ and Epstein-Barr virus $(\mathrm{EBV})^{19}$ infection have been recognized, the incidence of $\mathrm{HNC}$ has not decreased significantly in the USA, ${ }^{20}$ Asia, ${ }^{21,22}$ Europe $^{23}$ or Australia ${ }^{24}$ in recent years. Also, various kinds of treatment for these cancers are yet to be considered effective at eradicating the malignancies in case of late diagnosis. ${ }^{25,26}$ Regarding the incidence and mortality rate, the importance of prompt diagnosis cannot be overemphasized; still, a timely diagnosis of HNCs with the use of the current measures is yet to be achieved. ${ }^{27}$ Therefore, attention should be given to developing more efficient screening techniques and to their evaluation by the clinicians treating diseases of the head and neck region. ${ }^{28}$ However, the lack of a holistic account of DR HNCs precludes success in this matter.

\section{Objectives}

The recognition of the drugs which have been reported to cause HNCs and their documented presentations may help clinicians identify patients at risk so that the subsequent screening procedures and management of these patients may be accomplished without troublesome complications. We considered an analysis of publications about drugs that can potentially induce HNCs helpful; thus, the current descriptive study was undertaken to answer the following question: "Which drugs have been reported to induce cancers in the head and neck region or increase their risk?" by analyzing publications about DR HNCs.

\section{Material and methods}

Online databases, namely, PubMed, Embase, Cochrane Library, and Web of Science, were searched for relevant articles without any past date restrictions published until March 2020. The following keywords were used in the search queries: "head", "neck", "cancer", "malignancy", “drug”, "medication", "medicine”, and "medicament". Thesaurus terms, such as "therapyrelated cancer", "cancer of head and neck" and "head and neck neoplasms", were also used, according to the requirements of each database. In addition, journals with scope encompassing head and neck or oral oncology as well as the reference lists of relevant articles were also manually searched. Finally, complementary exploration was conducted in Google Scholar for articles related to the topic and those which cited studies relevant to drug-induced cancers. The obtained papers were screened by their titles and abstracts as well as by full texts in a stepwise manner. Eligible studies were determined as English-language case reports, case series, case-control studies, cohorts, randomized or non-randomized controlled studies, and longitudinal studies describing at least 1 drug which had resulted in $\mathrm{HNC}$ or had increased the risk of its development. Any studies with incomplete information, i.e., not mentioning the site of the newly developed cancer, the drug name or the type of cancer, were excluded. To exclude the outdated pharmacotherapeutic treatment protocols, we did not take into account studies published before January 2000. Finally, after a thorough search and screening process, 35 articles were included in this study. They comprised 30 case reports and case series, 3 longitudinal studies, 1 case-control study, and 1 cohort study. Table 1 presents a summary of the included studies sorted by the year of publication. Additionally, Fig. 1 depicts the flowchart of study selection and screening in detail.

The following data were extracted from the studies, where possible: author(s), year of publication, patients' age and gender, drug name and dosage, the primary disease for which the $\operatorname{drug}(\mathrm{s})$ were prescribed, type of cancer, cancer site, description of cases and control groups, odds ratios (ORs), and the significance of findings. To provide a better insight into the clinical characteristics of DR HNCs, we combined the data from all the included studies and analyzed them according to the assessed outcome, whenever possible. The assessed outcomes were age, gender, drug classification, drug name(s), additional drug(s), primary disorder(s), DR cancer, and its site. In case of absence of data in any of the studies regarding these outcomes, such study was excluded from the analysis. Table 2 presents the results of the combination of the data from all studies according to the aforementioned outcomes. 


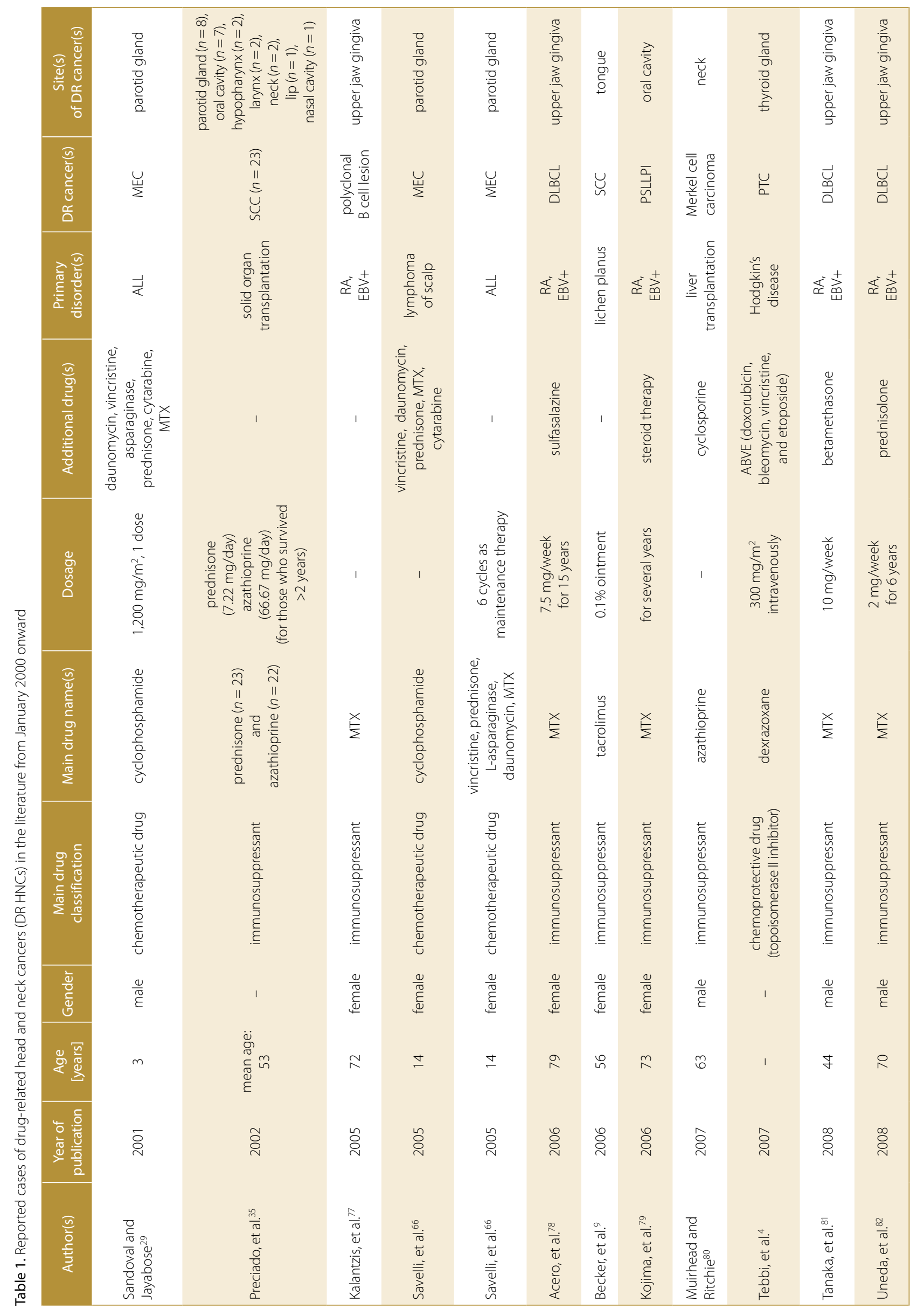




\begin{tabular}{|c|c|c|c|c|c|c|c|c|c|c|c|c|c|c|}
\hline 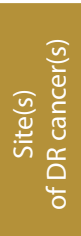 & 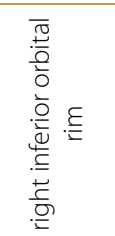 & 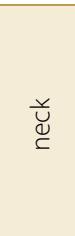 & 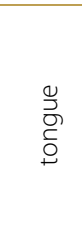 & $\stackrel{\varrho}{ }$ & 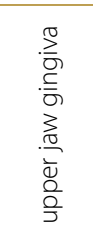 & 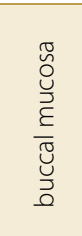 & $\begin{array}{l}\text { U. } \\
\text { Oे } \\
\text { D. }\end{array}$ & 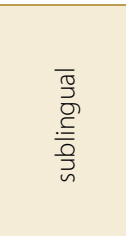 & 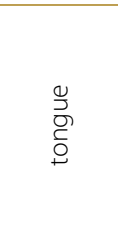 & 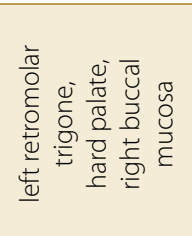 & $\begin{array}{l}\frac{\sqrt{0}}{\bar{x}} \\
\stackrel{\tilde{x}}{\varepsilon}\end{array}$ & 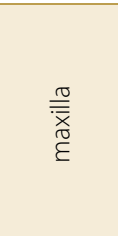 & $\cong$ & 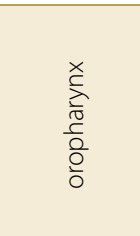 \\
\hline త & 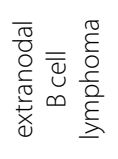 & 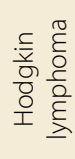 & $\stackrel{\bigcirc}{\Xi}$ & @ & 음 & 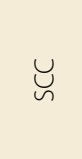 & 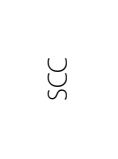 & 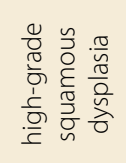 & $\breve{u}$ & 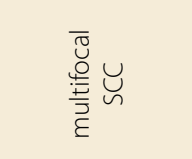 & 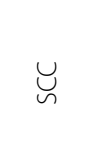 & 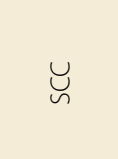 & $\underset{u}{u}$ & 음 \\
\hline 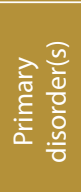 & $\mathbb{x}$ & 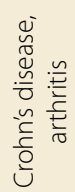 & 这总 & 达总 & 这总 & 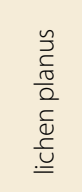 & 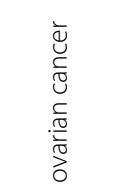 & 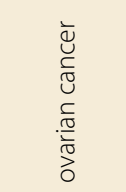 & 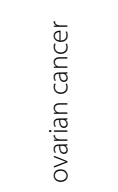 & 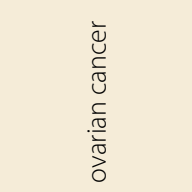 & 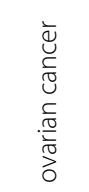 & 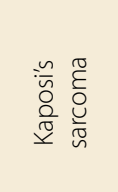 & 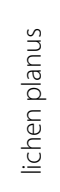 & $\overleftrightarrow{\propto}$ \\
\hline 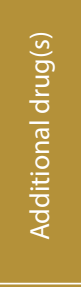 & 点 & $\stackrel{x}{\Sigma}$ & 1 & 1 & I & 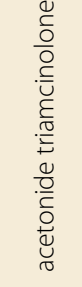 & 1 & 1 & 1 & 1 & 1 & 1 & 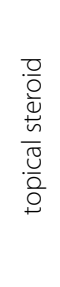 & 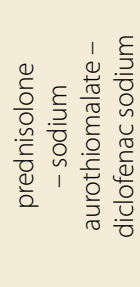 \\
\hline & 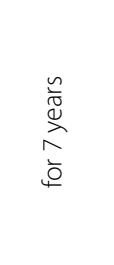 & 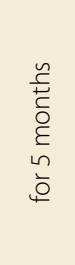 & 1 & 1 & 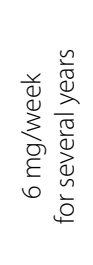 & 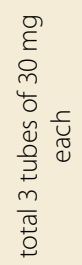 & 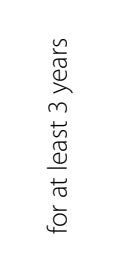 & 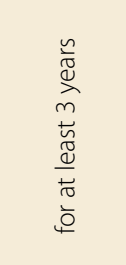 & 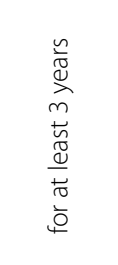 & 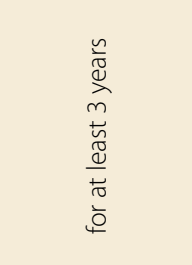 & 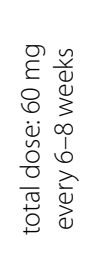 & 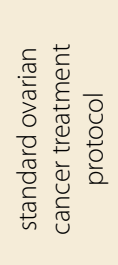 & $\begin{array}{l}\varepsilon \\
\stackrel{E}{\tilde{U}}\end{array}$ & 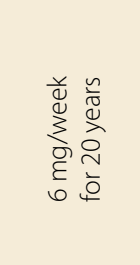 \\
\hline $\begin{array}{l}c \\
\frac{c}{q} \\
\frac{\pi}{c}\end{array}$ & 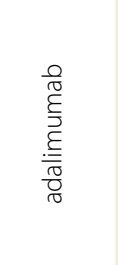 & 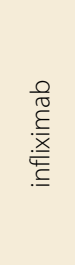 & $\stackrel{\times}{\Sigma}$ & $\stackrel{x}{\Sigma}$ & 茂 & 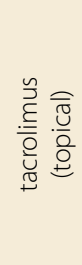 & 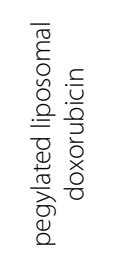 & 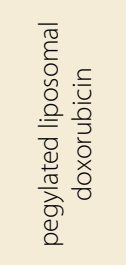 & 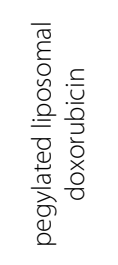 & 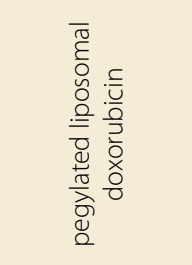 & $\begin{array}{l}: \frac{c}{0} \\
\frac{0}{0} \\
\frac{3}{2} \\
\frac{0}{0} \\
0\end{array}$ & $\begin{array}{l}: \frac{c}{0} \\
\frac{0}{0} \\
\frac{2}{0} \\
\frac{0}{0} \\
\frac{0}{0}\end{array}$ & 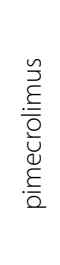 & $\stackrel{\times}{\Sigma}$ \\
\hline 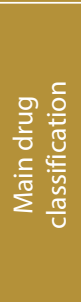 & 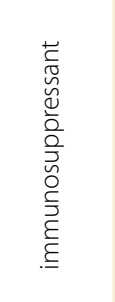 & 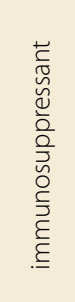 & 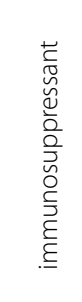 & 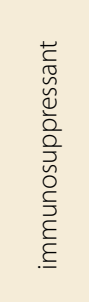 & 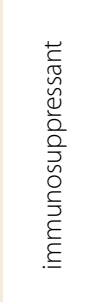 & 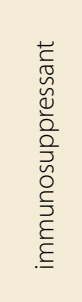 & 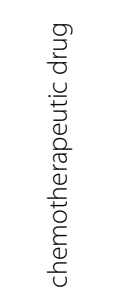 & 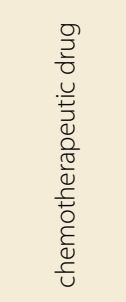 & 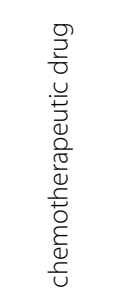 & 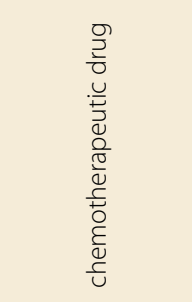 & 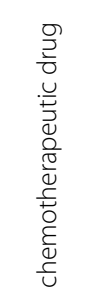 & 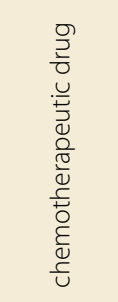 & 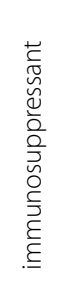 & 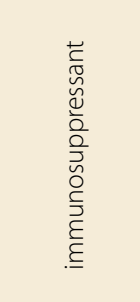 \\
\hline $\begin{array}{l}\frac{\bar{d}}{\mathrm{~g}} \\
\mathrm{~d} \\
\mathrm{~d}\end{array}$ & 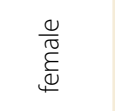 & 选 & $\frac{0}{\tilde{\Xi}}$ & 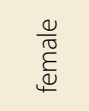 & 选 & $\frac{0}{\tilde{\Xi}}$ & 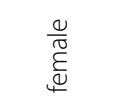 & 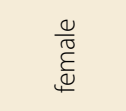 & 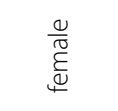 & 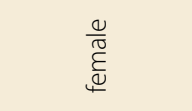 & 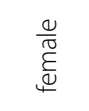 & 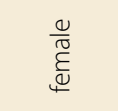 & $\frac{\stackrel{0}{\bar{\sigma}}}{\xi}$ & $\frac{\frac{\omega}{\tilde{\sigma}}}{\tilde{\varepsilon}}$ \\
\hline & $\approx$ & $\tilde{0}$ & $\infty$ & 8 & o & fo & 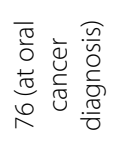 & 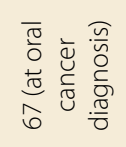 & 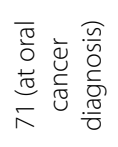 & 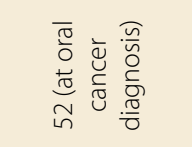 & in & in & in & 6 \\
\hline 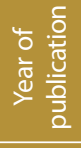 & ᄋ् & ळे & $\stackrel{\circ}{\circ}$ & 움 & $\stackrel{\circ}{\sim}$ & 윰 & $\overline{\bar{v}}$ & $\bar{\sim}$ & $\overline{\bar{N}}$ & $\overline{\bar{j}}$ & $\stackrel{m}{i}$ & $\stackrel{m}{\grave{n}}$ & $\stackrel{n}{\grave{\nu}}$ & $\stackrel{m}{\grave{n}}$ \\
\hline 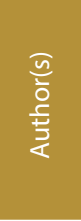 & 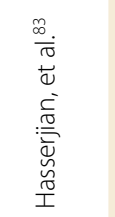 & 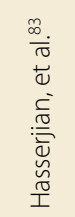 & 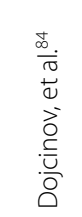 & 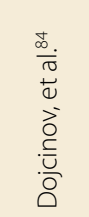 & 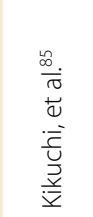 & 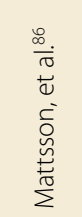 & 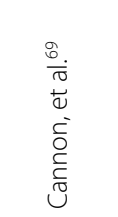 & 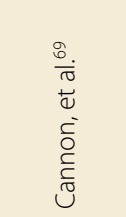 & 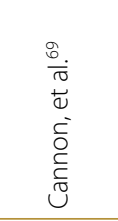 & 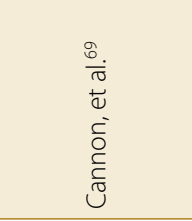 & 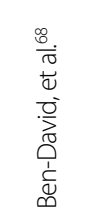 & 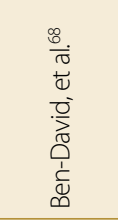 & 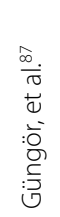 & 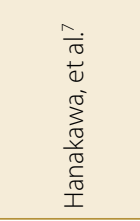 \\
\hline
\end{tabular}




\begin{tabular}{|c|c|c|c|c|c|c|c|c|c|c|c|c|c|}
\hline 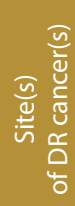 & $\begin{array}{l}\stackrel{0}{9} \\
\frac{5}{\sigma}\end{array}$ & 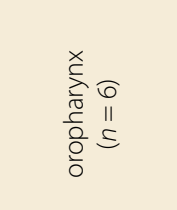 & 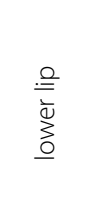 & 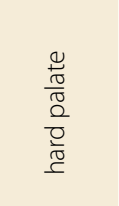 & 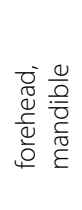 & 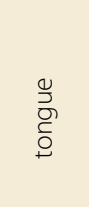 & 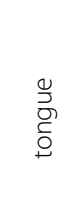 & 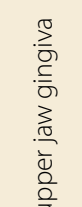 & $\begin{array}{l}\overline{0} \\
\frac{0}{0} \\
\frac{0}{\pi} \\
\frac{0}{0} \\
\frac{0}{0} \\
\frac{0}{0}\end{array}$ & $\begin{array}{l}\overline{0} \\
\frac{0}{0} \\
\frac{0}{01} \\
\frac{0}{0} \\
\frac{0}{0} \\
\frac{0}{0}\end{array}$ & 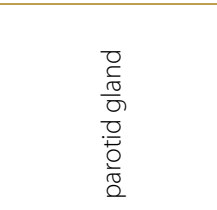 & $\begin{array}{l}\frac{0}{0} \\
\frac{0}{0} \\
\frac{0}{0} \\
\frac{0}{0} \\
\frac{0}{0} \\
\frac{0}{0}\end{array}$ & 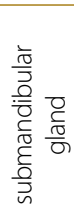 \\
\hline 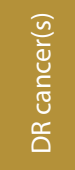 & $\stackrel{\partial}{a}$ & 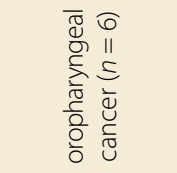 & 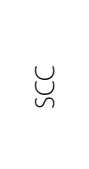 & $\begin{array}{l}\overrightarrow{0} \\
\overrightarrow{0}\end{array}$ & ্ֻ & @ & 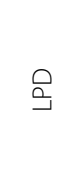 & $\begin{array}{l}\overrightarrow{\mathrm{O}} \\
\overrightarrow{0}\end{array}$ & $\stackrel{\vec{山}}{\Sigma}$ & $\stackrel{\breve{u}}{\Sigma}$ & $\stackrel{\vec{u}}{\Sigma}$ & $\stackrel{\breve{u}}{\Sigma}$ & 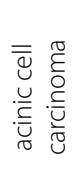 \\
\hline 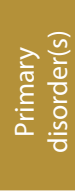 & 退䓵 & 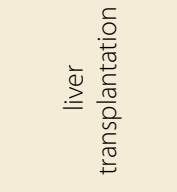 & 岕 & 压总 & 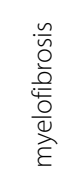 & 文怠 & 还总 & 遮总 & 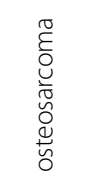 & 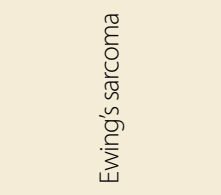 & 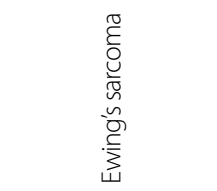 & 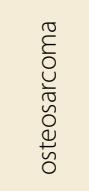 & 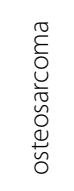 \\
\hline $\bar{\tau}$ & 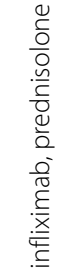 & 1 & 1 & 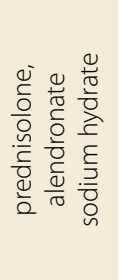 & 1 & 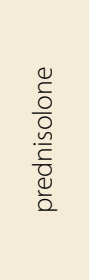 & 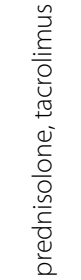 & 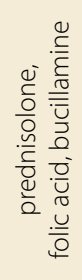 & 1 & 1 & 1 & 1 & 1 \\
\hline$\xi$ & 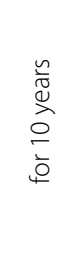 & 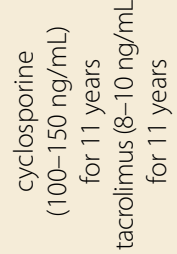 & 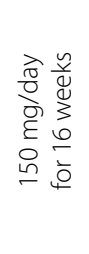 & 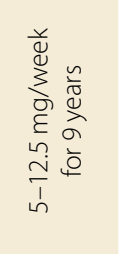 & 1 & 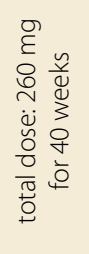 & 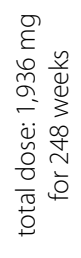 & 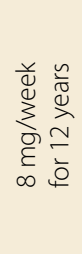 & 1 & 1 & 1 & 1 & 1 \\
\hline $\begin{array}{l}\frac{c}{0} \\
\frac{0}{2} \\
\frac{2}{0} \\
\frac{c}{\pi}\end{array}$ & 茂 & 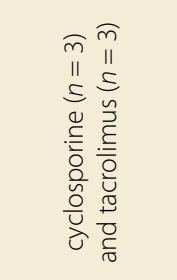 & 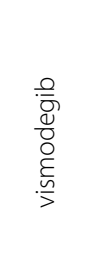 & $\stackrel{x}{\Sigma}$ & 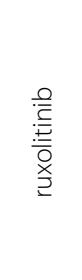 & $\frac{x}{\Sigma}$ & $\stackrel{\times}{\Sigma}$ & $\stackrel{x}{\Sigma}$ & 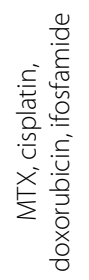 & 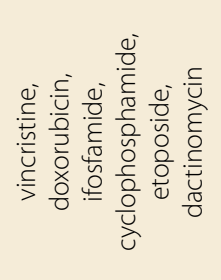 & 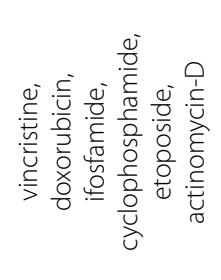 & 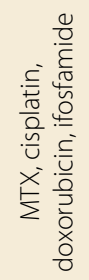 & 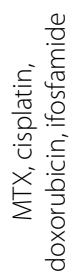 \\
\hline 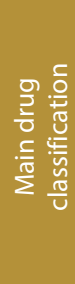 & 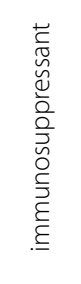 & 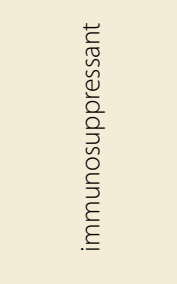 & 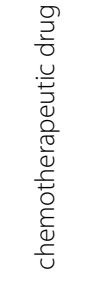 & 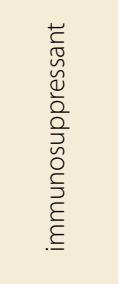 & 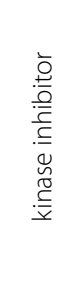 & 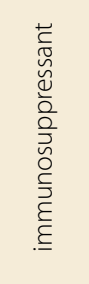 & 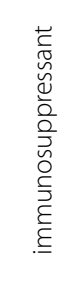 & 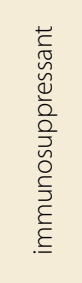 & 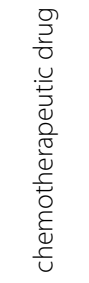 & 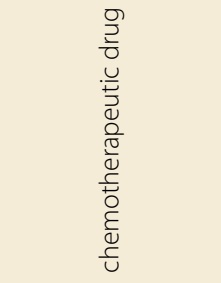 & 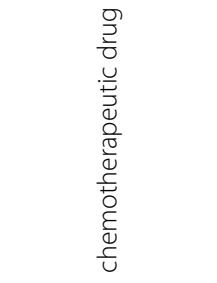 & 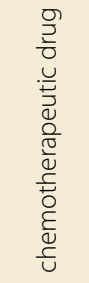 & 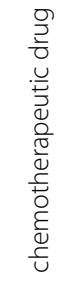 \\
\hline 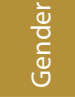 & $\begin{array}{l}\frac{\mathscr{U}}{\widetilde{\tilde{\Psi}}} \\
\underline{\underline{\Psi}}\end{array}$ & 1 & & 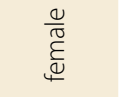 & $\underbrace{\frac{0}{\tilde{\Phi}}}$ & 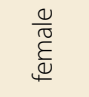 & 离 & $\frac{\stackrel{Q}{\sigma}}{\varepsilon}$ & $\frac{\Delta}{\stackrel{0}{\tilde{\varepsilon}}}$ & $\frac{0}{\tilde{\varepsilon}}$ & 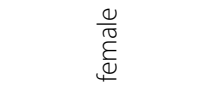 & $\frac{\Delta}{\stackrel{0}{\tilde{\varepsilon}}}$ & $\frac{\Delta}{\stackrel{\sigma}{\varepsilon}}$ \\
\hline$\frac{\square}{x}$ & $\stackrel{\circ}{\curvearrowright}$ & 1 & मे & $\hat{\sigma}$ & $\stackrel{+}{\perp}$ & 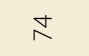 & $\stackrel{\nabla}{\curvearrowright}$ & 8 & $\underline{m}$ & $\underline{m}$ & $\circ$ & 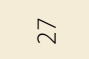 & $\approx$ \\
\hline 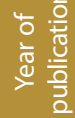 & $\bar{i}_{\bar{n}}^{m}$ & $\stackrel{m}{i}$ & $\underset{\sim}{\vec{N}}$ & $\stackrel{\vec{N}}{\sim}$ & $\grave{\sim}^{n}$ & $\stackrel{n}{\sim}$ & $\bar{\sim}$ & $\bar{\sim}$ & $\bar{\sim}$ & $\stackrel{n}{\bar{\sigma}}$ & $\stackrel{n}{\grave{n}}$ & 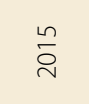 & $i^{n}$ \\
\hline$\stackrel{z}{Z}$ & 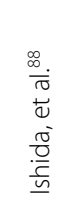 & 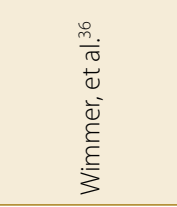 & 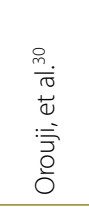 & 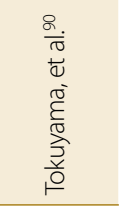 & 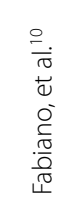 & 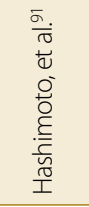 & 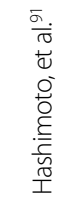 & 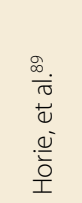 & 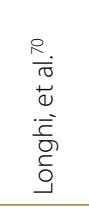 & 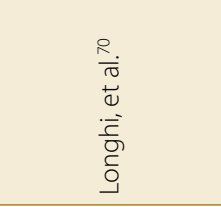 & 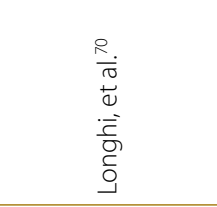 & 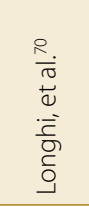 & 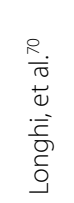 \\
\hline
\end{tabular}




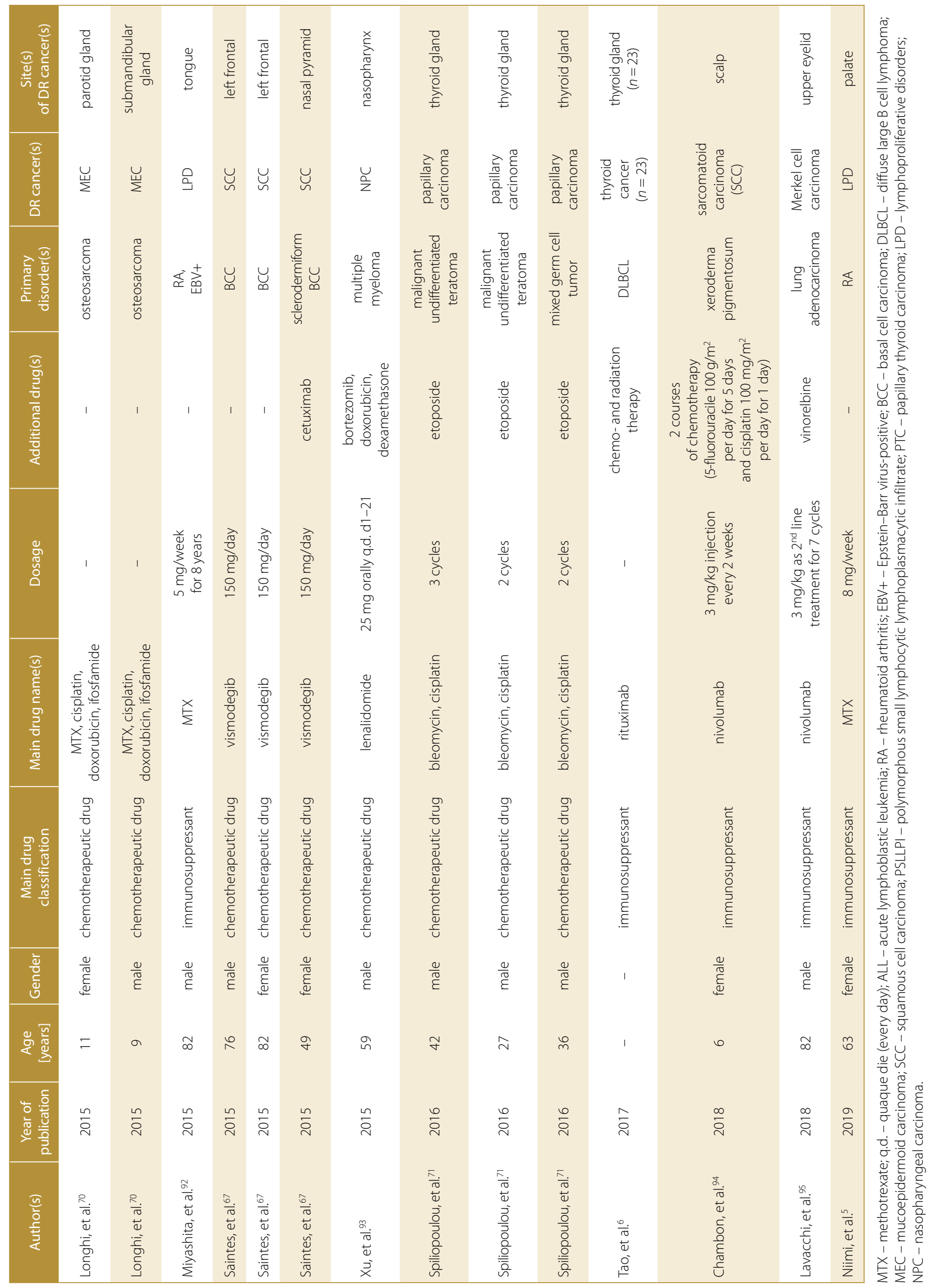




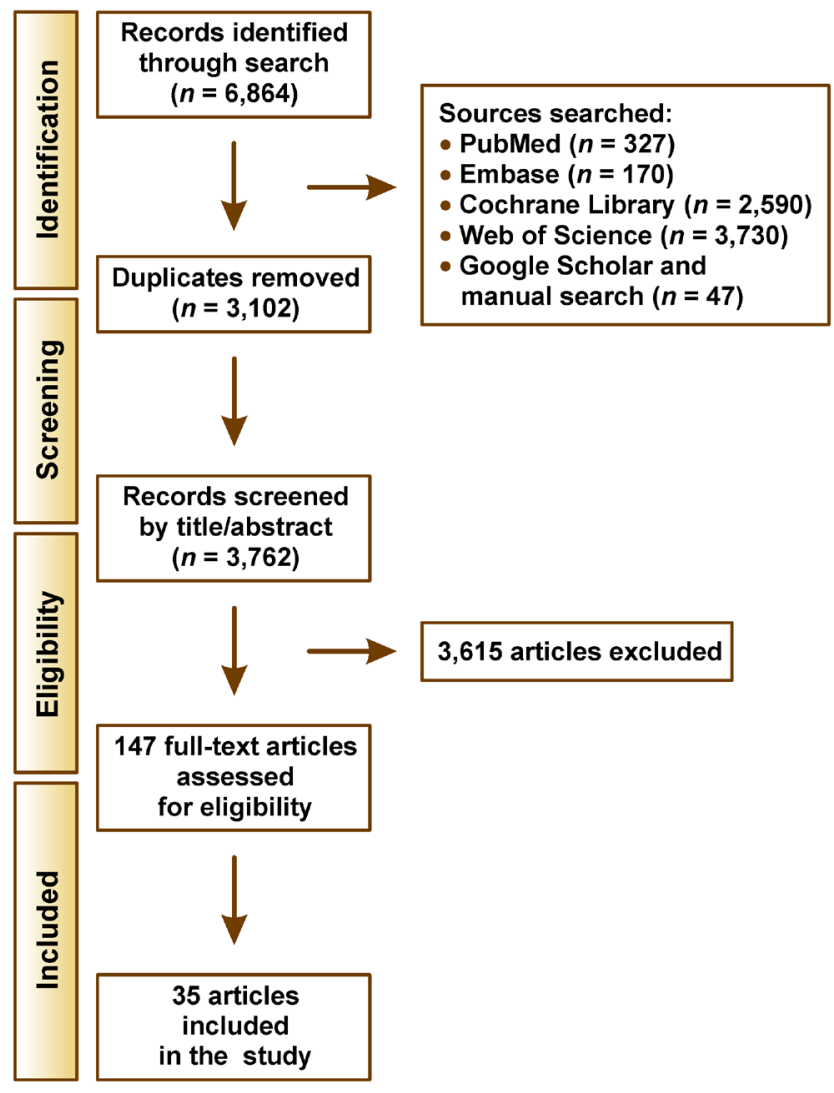

Fig. 1. Search flowchart

\section{Results and discussion}

We hereby discuss the most significant findings with regard to each outcome presented in Table 2.

\section{Age}

The lowest reported age for DR HNCs was 3 years, ${ }^{29}$ while the highest reported age was 84 years. ${ }^{30}$ Similar cancer incidences were found in the $1^{\text {st }}(<40$ years $)$ and $2^{\text {nd }}$ (40-60 years) age groups. However, the relative frequency of cancer incidence in the $3^{\text {rd }}$ group ( $>60$ years) was nearly twice as high as in other groups. This finding is in agreement with the latest statistics of the Surveillance, Epidemiology, and End Results (SEER) program, which also reports higher cancer rates in the older population. ${ }^{31}$ However, the relative frequencies of HNCs in the first 2 age groups in this program were much higher than those reported in the present survey. This possibly reflects the nature of DR cancers; drugs damage the genes related to the cancerassociated pathways, as opposed to the chronic accumulation of aberrant genetic and epigenetic changes, which are related to the pathogenesis of ordinary cancers. ${ }^{32,33}$ A potential implication of this finding, despite the limitations imposed by the type and number of studies, is that screening for DR HNCs should be implemented in a variety of age groups, conversely to the conventional screening procedures, which mainly screen the older-adult population. ${ }^{34}$ The mean age of patients at the time of diagnosis of DR HNCs was 46 years for males and 58 years for females. However, the mean age for the total number of cases of DR HNCs was 52.9 years. The difference between the mean age values for males, females and all cases in total could be explained by different total numbers of cases in each of these groups (21, 28 and 72, respectively). This difference between the numbers of the reported cases arose from the data inadequacy of some studies regarding the gender specifications of the HNC patients. ${ }^{4,6,35-37}$ The values of the reported mean age are lower than those reported for ordinary $\mathrm{HNCs}^{38}{ }^{38}$ which might hint at the role of drugs in inducing HNCs.

\section{Gender}

Although we attempted to classify the reported outcomes by gender specifications, we could not achieve this, as most of the data regarding gender were incomplete or ambiguous. Thus, the only outcome which could be classified by gender was the patients' age.

Regarding the total relative frequencies, the female/ male ratio of the diagnosed DR HNCs was $1.33 / 1$, which is contrary to the ratios reported for ordinary cancers, which occur more frequently in males than in females. ${ }^{38}$

Table 2. Summary of the data combined from the included studies regarding specific outcomes

\begin{tabular}{|c|c|c|c|c|}
\hline \multicolumn{3}{|c|}{ Specific outcome } & Frequency $(n)$ & Relative frequency* $[\%]$ \\
\hline \multirow{7}{*}{ Age [years] } & \multirow{2}{*}{$<40$} & male $29,70,71$ & 8 & 16.3 \\
\hline & & female $66,70,94$ & 5 & 10.2 \\
\hline & \multirow{2}{*}{$40-60$} & male $71,81,86,87,89,93$ & 6 & 12.2 \\
\hline & & female $9,67-69,84$ & 6 & 12.2 \\
\hline & \multirow{2}{*}{$>60$} & male $7,67,80,82,84,92,95$ & 7 & 14.3 \\
\hline & & female $5,10,30,67,69,77-79,83,85,88,90,91$ & 17 & 34.7 \\
\hline & total & & 49 & 100.0 \\
\hline \multirow{3}{*}{ Gender } & male & & 21 & 42.9 \\
\hline & female & & 28 & 57.1 \\
\hline & total & & 49 & 100.0 \\
\hline
\end{tabular}


A closer look at the data reveals that DR HNCs occurred in females slightly more often in the $2^{\text {nd }}$ than in the $1^{\text {st }}$ age group, as can be expected by taking account of the statistics of human papillomavirus (HPV)-negative HNCs. ${ }^{39}$ However, an interesting finding was that DR HNCs occurred approx. twice as often in females than males in the $3^{\text {rd }}$ age group. This finding may partly be explained by the shorter life expectancy of males in comparison with females, which results in a higher number of surviving senile females. ${ }^{40}$ Additionally, the most frequently used drug in this age group was methotrexate (MTX), which has been shown to incur higher acute toxicities in women than men, ${ }^{41}$ suggesting that sex-specific differences can also be a factor in determining adverse outcomes. Moreover, higher clearance rates of drugs such as ruxolitinib, ${ }^{42}$ doxorubicin $^{43}$ and adalimumab ${ }^{44}$ have been reported in males than females, which may contribute to increased morbidity in females. These findings could indicate that females may have a higher propensity for developing DR HNCs in older age, yet data on this matter remains limited.

\section{Main drugs (classification and names)}

Cancer-related drugs in the included studies could mainly be classified into 3 groups, namely, immunomodulators/immunosuppressants, and chemotherapeutic and chemoprotective drugs. The 5 most prevalent drugs in the immunomodulator/immunosuppressant group were corticosteroids, azathioprine, rituximab, MTX, and tacrolimus. The 5 most prevalent cancer-related drug prescriptions in the chemotherapeutic group were doxorubicin, cisplatin, ifosfamide, MTX, and cyclophosphamide. Only 1 study, in which dexrazoxane was used, evaluated the effects of chemoprotective drugs on cancer development. ${ }^{4}$

Predictably, carcinogenic potential has been shown for most of the aforementioned drugs, namely, azathioprine, ${ }^{45}$ cyclosporine, ${ }^{46}$ doxorubicin, ${ }^{47,48}$ cyclophosphamide ${ }^{49}$ cisplatin, ${ }^{50}$ ifosfamide, ${ }^{51}$ and dexrazoxane. ${ }^{52}$ However, carcinogenicity was not the only modality by which these drugs could have contributed to the development of secondary cancers. It has been shown that

\begin{tabular}{|c|c|c|c|c|}
\hline \multicolumn{3}{|c|}{ Specific outcome } & Frequency $(n)$ & Relative frequency* $[\%]$ \\
\hline \multirow{29}{*}{ Main drug classification and names } & \multirow{9}{*}{ immunosuppressant } & corticosteroid 35,66 & 24 & 15.4 \\
\hline & & azathioprine ${ }^{35,80}$ & 23 & 14.7 \\
\hline & & rituximab ${ }^{6}$ & 23 & 14.7 \\
\hline & & methotrexate $5,7,77-79,81,82,84,85,88-92$ & 16 & 10.3 \\
\hline & & tacrolimus $9,36,86$ & 5 & 3.2 \\
\hline & & cyclosporine ${ }^{36}$ & 3 & 1.9 \\
\hline & & nivolumab 94,95 & 2 & 1.3 \\
\hline & & pimecrolimus ${ }^{87}$ & 1 & 0.6 \\
\hline & & total & 97 & 62.2 \\
\hline & \multirow{18}{*}{ chemotherapeutic drug } & doxorubicin ${ }^{68-70}$ & 13 & 8.3 \\
\hline & & cisplatin 70,71 & 8 & 5.1 \\
\hline & & ifosfamide ${ }^{70}$ & 7 & 4.5 \\
\hline & & methotrexate ${ }^{66,70}$ & 6 & 3.8 \\
\hline & & cyclophosphamide $29,66,70$ & 4 & 2.6 \\
\hline & & vismodegib 30,67 & 4 & 2.6 \\
\hline & & bleomycin ${ }^{71}$ & 3 & 1.9 \\
\hline & & vincristine ${ }^{66,70}$ & 3 & 1.9 \\
\hline & & etoposide ${ }^{70}$ & 2 & 1.3 \\
\hline & & actinomycin-D 70 & 1 & 0.6 \\
\hline & & adalimumab ${ }^{83}$ & 1 & 0.6 \\
\hline & & dactinomycin ${ }^{70}$ & 1 & 0.6 \\
\hline & & daunomycin ${ }^{66}$ & 1 & 0.6 \\
\hline & & infliximab ${ }^{83}$ & 1 & 0.6 \\
\hline & & L-asparaginase ${ }^{66}$ & 1 & 0.6 \\
\hline & & lenalidomide ${ }^{93}$ & 1 & 0.6 \\
\hline & & ruxolitinib ${ }^{10}$ & 1 & 0.6 \\
\hline & & total & 58 & 37.1 \\
\hline & chemoprotective drug & dexrazoxane ${ }^{4}$ & 1 & 0.6 \\
\hline & \multicolumn{2}{|l|}{ total } & 156 & 99.9 \\
\hline
\end{tabular}


corticosteroids $^{53}$ and rituximab ${ }^{54,55}$ can inhibit the immune function, thus disrupting the normal immune system surveillance. Furthermore, it has been demonstrated that MTX, despite its immunosuppressive properties, is not associated with an increased cancer risk. ${ }^{56,57}$ However, it was the $4^{\text {th }}$ most frequently used drug in the immunodulator/immunosuppressant group. Furthermore, we found an additional controversy regarding the use of oral contraceptives. Although in a study by Grevers et al. the relationship between an increased thyroid cancer risk and the use of oral contraceptives was shown in the primary analysis, additional analyses proved that after adjusting for various other factors, their use was not associated with an increased thyroid cancer risk. ${ }^{58}$ Additionally, studies have shown that while the use of oral contraceptives can increase the risk of various cancers, ${ }^{59}$ their effects on thyroid cancer development are disputable. ${ }^{60-62}$ Due to inconsistent results regarding the use of oral contraceptives, we chose to exclude this type of drugs from our study. Such controversial findings warrant further research, yet it may be hypothesized that the many confounding variables which were present across the studies, e.g., age, predisposing conditions and co-carcinogens, might have contributed to DR HNCs development. Future research should describe the pathways by which these drugs may cause cancers, especially HNCs.

\section{Additional drugs}

As could be expected, additional drugs were similar to the main drugs analyzed in the included studies. The most prevalent additional drugs used in the studies were corticosteroids. The role of additional drugs in cancer development may be described as either facilitating cancer growth, as can be seen with the use of corticosteroids, or exacerbating cancer development, as can be observed with the use of cytotoxic drugs. Additionally, drug interactions might have also occurred in these studies, which could further contribute to the problem.

\section{Primary disorders}

From a clinical perspective, recognizing the conditions which may predispose a patient to secondary cancers, either due to their inherent characteristics

\begin{tabular}{|c|c|c|c|}
\hline & Specific outcome & Frequency $(n)$ & Relative frequency ${ }^{*}[\%]$ \\
\hline & corticosteroid 7,29,66,81,82,86-91,93 & 14 & 28.6 \\
\hline & methotrexate $29,66,83$ & 4 & 8.2 \\
\hline & etoposide ${ }^{4,71}$ & 4 & 8.2 \\
\hline & vincristine ${ }^{4,29,66}$ & 3 & 6.1 \\
\hline & cytarabine 29,66 & 2 & 4.1 \\
\hline & daunomycin 29,66 & 2 & 4.1 \\
\hline & doxorubicin 4,93 & 2 & 4.1 \\
\hline & acetonide $^{86}$ & 1 & 2.0 \\
\hline & alendronate sodium 90 & 1 & 2.0 \\
\hline & asparaginase 29 & 1 & 2.0 \\
\hline & bleomycin $^{4}$ & 1 & 2.0 \\
\hline & bortezomib ${ }^{93}$ & 1 & 2.0 \\
\hline Addlitionaldrus & bucillamine ${ }^{89}$ & 1 & 2.0 \\
\hline 9 & cetuximab ${ }^{67}$ & 1 & 2.0 \\
\hline & cisplatin ${ }^{94}$ & 1 & 2.0 \\
\hline & cyclosporine ${ }^{80}$ & 1 & 2.0 \\
\hline & diclofenac sodium ${ }^{7}$ & 1 & 2.0 \\
\hline & folic acid 89 & 1 & 2.0 \\
\hline & 5-fluorouracil ${ }^{94}$ & 1 & 2.0 \\
\hline & hydrate ${ }^{90}$ & 1 & 2.0 \\
\hline & infliximab ${ }^{88}$ & 1 & 2.0 \\
\hline & sodium aurothiomalate $^{7}$ & 1 & 2.0 \\
\hline & sulfasalazine ${ }^{78}$ & 1 & 2.0 \\
\hline & tacrolimus $^{91}$ & 1 & 2.0 \\
\hline & vinorelbine ${ }^{95}$ & 1 & 2.0 \\
\hline & total & 49 & 99.4 \\
\hline
\end{tabular}




\begin{tabular}{|c|c|c|c|}
\hline & Specific outcome & Frequency $(n)$ & Relative frequency* $[\%]$ \\
\hline \multirow{18}{*}{ Primary disorders } & organ transplantation $35,36,80$ & 30 & 25.4 \\
\hline & $\mathrm{LPD}^{* *} 4,6,66,93$ & 26 & 22.0 \\
\hline & rheumatoid arthritis $5,7,77-79,81-85,88,89,91,92$ & 17 & 14.4 \\
\hline & $\mathrm{EBV}+{ }^{77-79,81,82,84,85,88-92}$ & 14 & 11.9 \\
\hline & bone sarcoma ${ }^{70}$ & 7 & 5.9 \\
\hline & ovarian cancer 68,69 & 5 & 4.2 \\
\hline & basal cell carcinoma ${ }^{30,67}$ & 4 & 3.4 \\
\hline & lichen planus 9,86,87 & 3 & 2.5 \\
\hline & testicular cancer ${ }^{71}$ & 3 & 2.5 \\
\hline & leukemia 29,66 & 2 & 1.7 \\
\hline & actinic keratosis ${ }^{30}$ & 1 & 0.8 \\
\hline & arthritis 83 & 1 & 0.8 \\
\hline & Crohn's disease ${ }^{83}$ & 1 & 0.8 \\
\hline & lung adenocarcinoma ${ }^{95}$ & 1 & 0.8 \\
\hline & myelofibrosis ${ }^{10}$ & 1 & 0.8 \\
\hline & soft tissue sarcoma ${ }^{68}$ & 1 & 0.8 \\
\hline & xeroderma pigmentosum ${ }^{94}$ & 1 & 0.8 \\
\hline & total & 118 & 99.5 \\
\hline
\end{tabular}

or their pecific treatment, may be helpful when screening patients for DR HNCs. The 5 most prevalent conditions were organ transplantations, lymphoproliferative disorders (LPD), rheumatoid arthritis (RA), EBV infection, and bone sarcoma. As could be expected, these disorders were the predictors of the drug choices discussed in the previous sections. However, as the literature on DR HNCs is limited, clinicians should be aware of similar disorders when evaluating a patient. Additionally, screening by primary disorders may help with the diagnosis of more cases of $\mathrm{HNC}$, as different therapeutic regimens may not include the drugs which are listed in this study as cancer inducers.

\section{Drug-related cancers}

Although previous studies have omitted thyroid cancers and LPD from their HNC categories, ${ }^{63,64}$ we used the HNC definition provided by Holland et al. ${ }^{16}$ in order to perform a more comprehensive analysis. The 4 most prevalent types of DR HNCs were squamous cell carcinoma (SCC), thyroid cancer, LPD, and mucoepidermoid carcinoma/acinic cell carcinoma. Similar to ordinary thyroid cancers, ${ }^{65}$ the most prevalent cases of DR thyroid cancers were papillary carcinomas followed by follicular carcinomas. The relative frequencies of these DR cancers are in line with the results of previous studies, which reported higher prevalence for cancers of epithelial origin, ${ }^{63}$ although these studies excluded thyroid cancers from their analyses.

While the direct cause-and-effect relationship between drug use and cancer incidence cannot be established at this time, some findings can lead us to reflect on the role of drug therapy in cancer incidence. It has been stated in the literature that radiotherapy can increase the risk of secondary cancer in the head and neck region. ${ }^{66}$ While some patients in the included studies received radiotherapy in the head and neck

\begin{tabular}{|c|c|c|c|}
\hline & Specific outcome & Frequency $(n)$ & Relative frequency* [\%] \\
\hline \multirow{8}{*}{ DR cancers } & squamous cell carcinoma 9,10,30,35,67-69,86,87,94 & 38 & 36.9 \\
\hline & thyroid cancer ${ }^{4,6,71}$ & 27 & 26.2 \\
\hline & $\mathrm{LPD}^{* *} 5,7,77-79,81-85,88-92$ & 18 & 17.5 \\
\hline & mucoepidermoid carcinoma/acinic cell carcinoma $29,66,70$ & 10 & 9.7 \\
\hline & pharyngeal cancer ${ }^{36,93}$ & 7 & 6.8 \\
\hline & Merkel cell carcinoma ${ }^{80,95}$ & 2 & 1.9 \\
\hline & sarcomatoid carcinoma ${ }^{94}$ & 1 & 0.9 \\
\hline & total & 103 & 99.9 \\
\hline
\end{tabular}


region, ${ }^{67-69}$ others did not receive it, ${ }^{10,29,30,70-73}$ or reported that HNCs had developed regardless of receiving or not receiving radiotherapy. ${ }^{6}$ While the genetic or acquired predisposition of individuals to secondary cancers through field cancerization, ${ }^{74}$ immunosuppression, ${ }^{75}$ or even infection with oncogenic viruses ${ }^{76}$ cannot be ignored, the temporal relationship between drug use and cancer incidence, which was sometimes observed within months of its use ${ }^{30}$ may indeed indicate that drug use can have a significant impact on HNC development.

To explain the biological plausibility of this statement, the most prevalent drug classifications used in the included studies should be considered. The 2 most often mentioned cancer-related drug classes in these studies were immunomodulatory/immunosuppressive and chemotherapeutic drugs. These drugs may induce the double-strand breakage of the DNA structure, which may result in aberrant lesions. ${ }^{77}$ Additionally, immunosuppression can impair immune surveillance, ${ }^{75}$ as some chemotherapeutic drugs do. ${ }^{66}$ However, the exact mechanisms through which drugs can cause HNCs is not yet clear.

Other, less prevalent DR HNCs reported in the literature were pharyngeal cancers, Merkel cell carcinoma and sarcomatoid carcinoma. Additional entities of DR HNCs may also be present, but they have not yet been discovered. Clinicians should be aware of the possibility of occurrence of these DR HNCs to diagnose them in a timely manner.

\section{Site of drug-related cancers}

The sites of DR HNCs closely correspond to the types of DR HNCs discussed in the previous section. These sites were the oral cavity, neck, salivary glands, including the parotid and submandibular glands, pharynx/ larynx, and head/face. Clinicians should take note of the most prevalent sites of DR HNCs and implement appropriate diagnostic screening measures, such as the continuous surveillance of each site.

\section{Limitations}

Although we tried to find as many studies as possible about the subject matter, and analyze their data in a way that could be easily comprehended, this study was not without limitations. Most of the included studies about DR HNCs were case reports or cases series, which inevitably have a low level of evidence. Furthermore, some of the studies' data regarding the analyzed outcomes were incomplete. We hope that this descriptive study will help clinicians, including dentists and general practitioners, in diagnosing HNC patients. A detailed discussion on the possible intra- and extracellular pathways responsible for the development and promotion of DR cancers was well outside the scope of this study. Future research should not only be dedicated to DR HNCs, but also to DR cancers occurring within other parts of the human body.

\section{Conclusions}

In summary, drug therapy may induce secondary cancers in the head and neck region. These DR HNCs were more prevalent in older-adult populations, with no noticeable disparities between males and females after adjusting for sexspecific drugs. The most common DR HNCs were thyroid cancer, SCC, LPD, and mucoepidermoid carcinoma/acinic cell carcinoma, with the neck being the most commonly affected site. Drugs that can cause these types of cancers can be broadly classified into chemotherapeutic drugs, immunomodulatory/immunosuppressive drugs, oral contraceptives, and chemoprotective drugs. Although biologically plausible pathways may be hypothesized as the mechanisms of action of these drugs in inducing DR HNCs, the direct relationship between drug use and HNCs is yet to be established. Further research is necessary to understand the nature of DR HNCs.

\section{ORCID iDs}

Hamed Mortazavi (D) https://orcid.org/0000-0002-0778-5299 Behrad Rahbani Nobar (D) https://orcid.org/0000-0002-4230-3590

Shervin Shafiei (D) https://orcid.org/0000-0002-0415-4678 Nima Ahmadi (D) https://orcid.org/0000-0002-8685-6058

\begin{tabular}{|c|c|c|c|}
\hline & Specific outcome & Frequency $(n)$ & Relative frequency* $[\%]$ \\
\hline \multirow{6}{*}{ Sites of DR cancers ${ }^{* * *}$} & oral cavity $5,9,30,69,77-79,81,82,84-92$ & 33 & 31.4 \\
\hline & neck $^{4,6,35,71,80,83}$ & 31 & 29.5 \\
\hline & salivary glands $29,35,66,70$ & 18 & 17.1 \\
\hline & pharynx/larynx $7,35,36,93$ & 12 & 11.4 \\
\hline & head/face $10,35,67,68,83,94,95$ & 11 & 10.2 \\
\hline & total & 105 & 99.6 \\
\hline
\end{tabular}

* Note that the sum of the relative frequencies may not equal to $100 \%$ due to the rounding of numbers. ${ }^{*}$ LPD includes the following: Hodgkin and non-Hodgkin lymphomas; DLBCL; multiple myeloma; polyclonal B cell lesion; pseudolymphoma; and PSLLPI. *** Details of each site: oral cavity - lips, tongue, gingiva, palate, buccal mucosa, alveolar ridge, retromolar trigone, and sublingual; neck - thyroid gland, cervical lymph node and soft tissue; salivary glands - parotid and submandibular glands; pharynx/larynx - nasopharynx, oropharynx, hypopharynx, and larynx; head/face - scalp, frontal, zygoma, ethmoid sinus, orbital rim, maxilla, mandible, ear, eyelid, chin, nasal cavity, and pyramid. 


\section{References}

1. Schmähl D, Habs M. Drug-induced cancer. In: Grundmann E, ed. Drug-Induced Pathology. Berlin-Heidelberg, Germany: SpringerVerlag; 1980:333-369.

2. Hoover R, Fraumeni JF Jr. Drug-induced cancer. Cancer. 1981;47(5 Suppl):1071-1080. doi:10.1002/1097-0142(19810301)47:5+ $<1071$ ::aid-cncr2820471304>3.0.co;2-7

3. Fraumeni JF Jr. Drug-induced cancer. J Natl Cancer Inst. 1972;48(5):1267-1270. doi:10.1093/jnci/48.5.1267

4. Tebbi CK, London WB, Friedman D, et al. Dexrazoxane-associated risk for acute myeloid leukemia/myelodysplastic syndrome and other secondary malignancies in pediatric Hodgkin's disease. J Clin Oncol. 2007:25(5):493-500. doi:10.1200/JCO.2005.02.3879

5. Niimi K, Shingaki S, Funayama A, et al. Oral and maxillofacial manifestations of methotrexate-associated lymphoproliferative disorder in a patient with rheumatoid arthritis: Report of a case. J Oral Maxillofac Surg Med Pathol. 2019;31(2):86-93. doi:10.1016/j.ajoms.2018.07.010

6. Tao L, Clarke CA, Rosenberg AS, et al. Subsequent primary malignancies after diffuse large B-cell lymphoma in the modern treatment era. Br J Heamatol. 2017;178(1):72-80. doi:10.1111/bjh.14638

7. Hanakawa H, Orita Y, Sato Y, Uno K, Nishizaki K, Yoshino T. Large ulceration of the oropharynx induced by methotrexate-associated lymphoproliferative disorders. Acta Med Okayama. 2013;67(4):265-269. doi:10.18926/AMO/51072

8. Cunningham F, Darling MI, Gangopadhyay M, Jackson R, McKay P, Bilsland D. An isolated ulcerated forehead lesion in a 70-year-old man. Clin Exp Dermatol. 2017;42(5):554-557. doi:10.1111/ced.13093

9. Becker JC, Houben R, Vetter CS, Bröcker EB. The carcinogenic potential of tacrolimus ointment beyond immune suppression: $A$ hypothesis creating case report. BMC Cancer. 2006;6:7. doi:10.1186/1471-2407-6-7

10. Fabiano $A$, Calzavara-Pinton $P$, Monari $P$, et al. Eruptive squamous cell carcinomas with keratoacanthoma-like features in a patient treated with ruxolitinib. Br J Dermatol. 2015;173(4):1098-1099. doi:10.1111/bjd.13922

11. Treyve EL, Duckert LG. Phenytoin-induced lymphadenopathy appearing as a nasopharyngeal malignant neoplasm. Arch Otolaryngol. 1981;107(6):382-384. doi:10.1001/archotol.1981.00790420056012

12. Knight A, Bailey J, Balcombe J, Farland WH, Rodan B, Preuss P. Which drugs cause cancer? For and against: Cancer bioassays. BMJ. 2005;331(7521):E389-E391. doi:10.1136/bmj.331.7521.E389

13. Hartung T. Toxicology for the twenty-first century. Nature. 2009:460(7252):208-212. doi:10.1038/460208a

14. Ward JM. The two-year rodent carcinogenesis bioassay - will it survive? J Toxicol Pathol. 2007;20(1):13-19. doi:10.1293/tox.20.13

15. Yuan A, Woo SB. Adverse drug events in the oral cavity. Oral Surg Oral Med Oral Pathol Oral Radiol. 2015;119(1):35-47. doi:10.1016/j.0ooo.2014.09.009

16. Holland JF. Cancer Medicine (Two-Volume Set). $4^{\text {th }}$ ed. Beijing, China: PMPH USA, Ltd.; 1999.

17. Bray F, Ferlay J, Soerjomataram I, Siegel RL, Torre LA, Jemal A. Global cancer statistics 2018: GLOBOCAN estimates of incidence and mortality worldwide for 36 cancers in 185 countries. CA Cancer J Clin. 2018;68(6):394-424. doi:10.3322/caac.21492

18. Shaw R, Beasley N. Aetiology and risk factors for head and neck cancer: United Kingdom National Multidisciplinary Guidelines. J Laryngol Otol. 2016;130(S2):S9-S12. doi:10.1017/S0022215116000360

19. Pezzuto F, Buonaguro L, Caponigro F, et al. Update on head and neck cancer: Current knowledge on epidemiology, risk factors, molecular features and novel therapies. Oncology. 2015;89(3):125-136. doi:10.1159/000381717

20. Mourad M, Jetmore T, Jategaonkar AA, Moubayed S, Moshier E, Urken ML. Epidemiological trends of head and neck cancer in the United States: A SEER population study. J Oral Maxillofac Surg. 2017;75(12):2562-2572. doi:10.1016/j.joms.2017.05.008

21. Mishra A, Meherotra R. Head and neck cancer: Global burden and regional trends in India. Asian Pac J Cancer Prev. 2014;15(2):537-550. doi:10.7314/apjcp.2014.15.2.537

22. Mirzaei M, Hosseini SA, Ghoncheh M, et al. Epidemiology and trend of head and neck cancers in Iran. Glob J Health Sci. 2016;8(1):189-193. doi:10.5539/gjhs.v8n1p189

23. Döbrossy L. Epidemiology of head and neck cancer: Magnitude of the problem. Cancer Metastasis Rev. 2005;24(1):9-17. doi:10.1007/ s10555-005-5044-4
24. Ariyawardana A, Johnson NW. Trends of lip, oral cavity and oropharyngeal cancers in Australia 1982-2008: Overall good news but with rising rates in the oropharynx. BMC Cancer. 2013;13:333. doi:10.1186/1471-2407-13-333

25. Coca-Pelaz A, Takes RP, Hutcheson $K$, et al. Head and neck cancer: A review of the impact of treatment delay on outcome. Adv Ther. 2018;35(2):153-160. doi:10.1007/s12325-018-0663-7

26. Douglas CM, Ingarfield K, McMahon AD, Savage SA, Conway DI, MacKenzie K. Presenting symptoms and long-term survival in head and neck cancer. Clin Otolaryngol. 2018;43(3):795-804. doi:10.1111/coa.13053

27. Gerstner $\mathrm{AOH}$. Early detection in head and neck cancer - current state and future perspectives. GMS Curr Top Otorhinolaryngol Head Neck Surg. 2008;7:Doc06.

28. Hashim D, Genden E, Posner M, Hashibe M, Boffetta P. Head and neck cancer prevention: From primary prevention to impact of clinicians on reducing burden. Ann Oncol. 2019;30(5):744-756. doi:10.1093/annonc/mdz084

29. Sandoval C, Jayabose S. Parotid mucoepidermoid carcinoma following chemotherapy for childhood acute lymphoblastic leukemia. Pediatric Hematol Oncol. 2001;18(3):217-220. doi:10.1080/08880010151114903

30. Orouji A, Goerdt S, Utikal J, Leverkus M. Multiple highly and moderately differentiated squamous cell carcinomas of the skin during vismodegib treatment of inoperable basal cell carcinoma. Br J Dermatol. 2014;171(2):431-433. doi:10.1111/bjd.12840

31. Siegel RL, Miller KD, Jemal A. Cancer statistics, 2020. CA Cancer J Clin. 2020;70(1):7-30. doi:10.3322/caac.21590

32. Hanahan D, Weinberg RA. The hallmarks of cancer. Cell. 2000;100(1):57-70. doi:10.1016/s0092-8674(00)81683-9

33. Hanahan D, Weinberg RA. Hallmarks of cancer: The next generation. Cell. 2011;144(5):646-674. doi:10.1016/j.cell.2011.02.013

34. Smith RA, Andrews KS, Brooks D, et al. Cancer screening in the United States, 2019: A review of current American Cancer Society guidelines and current issues in cancer screening. CA Cancer J Clin. 2019;69(3):184-210. doi:10.3322/caac.21557

35. Preciado DA, Matas A, Adams GL. Squamous cell carcinoma of the head and neck in solid organ transplant recipients. Head Neck. 2002;24(4):319-325. doi:10.1002/hed.10055

36. Wimmer CD, Angele MK, Schwarz B, et al. Impact of cyclosporine versus tacrolimus on the incidence of de novo malignancy following liver transplantation: A single center experience with 609 patients. Transpl Int. 2013;26(10):999-1006. doi:10.1111/tri.12165

37. Vigneswaran N, Williams MD. Epidemiologic trends in head and neck cancer and aids in diagnosis. Oral Maxillofac Surg Clin North Am. 2014;26(2):123-141. doi:10.1016/j.coms.2014.01.001

38. Rettig EM, D'Souza G. Epidemiology of head and neck cancer. Surg Oncol Clin N Am. 2015;24(3):379-396. doi:10.1016/j.soc.2015.03.001

39. Kochanek KD, Murphy SL, Xu J, Arias E. Deaths: Final data for 2017. Natl Vital Stat Rep. 2019;68(9):1-77.

40. Hoekstra $M$, van Ede $A E$, Haagsma $C$, et al. Factors associated with toxicity, final dose, and efficacy of methotrexate in patients with rheumatoid arthritis. Ann Rheum Dis. 2003;62(5):423-426. doi:10.1136/ard.62.5.423

41. Chen X, Williams WV, Sandor V, Yeleswaram S. Population pharmacokinetic analysis of orally-administered ruxolitinib (INCB018424 phosphate) in patients with primary myelofibrosis (PMF), postpolycythemia vera myelofibrosis (PPV-MF) or post-essential thrombocythemia myelofibrosis (PET MF). J Clin Pharmacol. 2013;53(7):721-730. doi:10.1002/jcph.102

42. Dobbs NA, Twelves CJ, Gillies H, James CA, Harper PG, Rubens RD. Gender affects doxorubicin pharmacokinetics in patients with normal liver biochemistry. Cancer Chemother Pharmacol. 1995;36(6):473-476. doi:10.1007/BF00685796

43. Ternant D, Ducourau E, Fuzibet $P$, et al. Pharmacokinetics and concentration-effect relationship of adalimumab in rheumatoid arthritis. Br J Clin Pharmacol. 2015;79(2):286-297. doi:10.1111/bcp.12509

44. Kinlen LJ, Sheil AG, Peto J, Doll R. Collaborative United Kingdom-Australasian study of cancer in patients treated with immunosuppressive drugs. Br Med J. 1979;2(6203):1461-1466. doi:10.1136/bmj.2.6203.1461 
45. Olshan AF, Mattison DR, Zwanenburg TSB. Cyclosporine A: Review of genotoxicity and potential for adverse human reproductive and developmental effects: Report of a working group on the genotoxicity of cyclosporine A, August 18, 1993. Mutat Res Rev Genet Toxicol. 1994;317(2):163-173. doi:10.1016/0165-1110(94)90023-X

46. Potter AJ, Gollahon KA, Palanca BJA, et al. Flow cytometric analysis of the cell cycle phase specificity of DNA damage induced by radiation, hydrogen peroxide and doxorubicin. Carcinogenesis. 2002;23(3):389-401. doi:10.1093/carcin/23.3.389

47. Chambers SK, Chopyk RL, Chambers JT, Schwartz PE, Duffy TP. Development of leukemia after doxorubicin and cisplatin treatment for ovarian cancer. Cancer. 1989;64(12):2459-2461. doi:10.1002/10970142(19891215)64:12<2459::aid-cncr2820641210>3.0.co;2-t

48. van den Brand JAJG, van Dijk PR, Hofstra JM, Wetzels JFM. Cancer risk after cyclophosphamide treatment in idiopathic membranous nephropathy. Clin J Am Soc Nephrol. 2014;9(6):1066-1073. doi:10.2215/CJN.08880813

49. Greene $\mathrm{MH}$. Is cisplatin a human carcinogen? J Natl Cancer Inst. 1992;84(5):306-312. doi:10.1093/jnci/84.5.306

50. Sannu A, Radha R, Mathews A, Padmakumari Mony R, Prahladan A, James FV. Ifosfamide-induced malignancy of ureter and bladder. Cureus. 2017;9(8):e1594. doi:10.7759/cureus.1594

51. Friedrich A, Olejniczak K. Evaluation of carcinogenicity studies of medicinal products for human use authorised via the European centralised procedure (1995-2009). Regul Toxicol Pharmacol. 2011;60(2):225-248. doi:10.1016/j.yrtph.2011.04.001

52. Maxwell R, Luksik AS, Garzon-Muvdi T, et al. Contrasting impact of corticosteroids on anti-PD-1 immunotherapy efficacy for tumor histologies located within or outside the central nervous system. Oncoimmunology. 2018;7(12):e1500108. doi:10.1080/216 2402X.2018.1500108

53. Tan TT, Coussens LM. Humoral immunity, inflammation and cancer. Curr Opin Immunol. 2007;19(2):209-216. doi:10.1016/j.coi.2007.01.001

54. Aksoy S, Arslan C, Harputluoglu H, Dizdar O, Altundag K. Malignancies after rituximab treatment: Just coincidence or more? J BUON. 2011;16(1):112-115.

55. Hoffmeister RT. Methotrexate therapy in rheumatoid arthritis: 15 years experience. Am J Med. 1983;75(6A):69-73. doi:10.1016/0002-9343(83)90477-1

56. Turnbull C, Roach M. Is methotrexate carcinogenic? Br Med J. 1980;281(6243):808. doi:10.1136/bmj.281.6243.808-c

57. Zivaljevic V, Vlajinac $H$, Jankovic $R$, et al. Case-control study of female thyroid cancer - menstrual, reproductive and hormonal factors. Eur J Cancer Prev. 2003;12(1):63-66. doi:10.1097/00008469-200302000-00010

58. Grevers $X$, Grundy A, Poirier $A E$, et al. Cancer incidence attributable to the use of oral contraceptives and hormone therapy in Alberta in 2012. CMAJ Open. 2016;4(4):E754-E759. doi:10.9778/cmajo.20160046

59. Ron E, Kleinerman RA, Boice JD Jr., LiVolsi VA, Flannery JT, Fraumeni JF Jr. A population-based case-control study of thyroid cancer. J Natl Cancer Inst. 1987;79(1):1-12.

60. Wu L, Zhu J. Linear reduction in thyroid cancer risk by oral contraceptive use: A dose-response meta-analysis of prospective cohort studies. Hum Reprod. 2015;30(9):2234-2240. doi:10.1093/humrep/dev160

61. La Vecchia C, Ron E, Franceschi S, et al. A pooled analysis of casecontrol studies of thyroid cancer. III. Oral contraceptives, menopausal replacement therapy and other female hormones. Cancer Causes Control. 1999;10(2):157-166. doi:10.1023/a:1008832513932

62. Cohen N, Fedewa S, Chen AY. Epidemiology and demographics of the head and neck cancer population. Oral Maxillofac Surg Clin North Am. 2018;30(4):381-395. doi:10.1016/j.coms.2018.06.001

63. Stoyanov GS, Kitanova M, Dzhenkov DL, Ghenev P, Sapundzhiev N. Demographics of head and neck cancer patients: A single institution experience. Cureus. 2017;9(7):e1418. doi:10.7759/cureus.1418

64. Cabanillas ME, McFadden DG, Durante C. Thyroid cancer. Lancet. 2016;388(10061):2783-2795. doi:10.1016/S0140-6736(16)30172-6

65. Ng SP, Pollard C III, Kamal M, et al. Risk of second primary malignancies in head and neck cancer patients treated with definitive radiotherapy. NPJ Precis Oncol. 2019;3:22. doi:10.1038/s41698-019-0097-y

66. Savelli SL, Klopfenstein KJ, Termuhlen AM. Mucoepidermoid carcinoma of the parotid gland as a second malignant neoplasm. Pediatr Blood Cancer. 2005;45(7):997-1000. doi:10.1002/pbc.20306
67. Saintes C, Saint-Jean M, Brocard A, et al. Development of squamous cell carcinoma into basal cell carcinoma under treatment with vismodegib. J Eur Acad Dermatol Venereol. 2015;29(5):1006-1009. doi:10.1111/jdv.12526

68. Ben-David Y, Leiser Y, Kachta O, El-Naaj IA. Does long-term treatment with Doxil ${ }^{\circledR}$ predispose patients to oral cancer? Int J Clin Oncol. 2013;18(3):554-555. doi:10.1007/s10147-012-0400-1

69. Cannon TL, Muggia F, Hirsch D, Andreopoulou EA, Kerr AR, DeLacure MD. Multiple cases of squamous cell carcinoma of the tongue and oral cavity in patients treated with long-term pegylated liposomal doxorubicin (PLD) for ovarian cancer. J Clin Oncol. 2011;29(15 Suppl):5557. doi:10.1200/jco.2011.29.15_suppl.5557

70. Longhi A, Errani C, Gambarotti M, et al. Salivary gland second cancer after bone sarcoma treatment. Eur J Orthop Surg Traumatol. 2015;25(7):1201-1204. doi:10.1007/s00590-015-1662-x

71. Spiliopoulou P, Bowers SP, Gibson S, White J, Reed N. Three cases of thyroid cancer following the diagnosis of testicular cancer: Treatment-related complication or genetics? Scott Med J. 2016;61(2):111-116. doi:10.1177/0036933016635409

72. Jaiswal G, Jaiswal S, Kumar R, Sharma A. Field cancerization: Concept and clinical implications in head and neck squamous cell carcinoma. J Exp Ther Oncol. 2013;10(3):209-214.

73. Gutierrez-Dalmau A, Campistol JM. Immunosuppressive therapy and malignancy in organ transplant recipients: A systematic review. Drugs. 2007;67(8):1167-1198. doi:10.2165/00003495-200767080-00006

74. Chang Y, Moore PS, Weiss RA. Human oncogenic viruses: Nature and discovery. Philos Trans R Soc Lond B Biol Sci. 2017;372(1732):20160264. doi:10.1098/rstb.2016.0264

75. Krishnan B, Morgan GJ. Non-Hodgkin lymphoma secondary to cancer chemotherapy. Cancer Epidemiol Biomarkers Prev. 2007;16(3):377-380. doi:10.1158/1055-9965.EPI-06-1069

76. Hipp MM, Hilf N, Walter S, et al. Sorafenib, but not sunitinib, affects function of dendritic cells and induction of primary immune responses. Blood. 2008;111(12):5610-5620. doi:10.1182/blood-2007-02-075945

77. Kalantzis A, Marshman Z, Falconer DT, Morgan PR, Odell EW. Oral effects of low-dose methotrexate treatment. Oral Surg Oral Med Oral Pathol Oral Radiol Endod. 2005;100(1):52-62. doi:10.1016/j.tripleo.2004.08.020

78. Acero J, Navarro-Cuellar C, Menarguez J, Herencia H, Navarro-Vila C. Naso-maxillary non-Hodgkin lymphoma associated with methotrexate treatment in a patient with rheumatoid arthritis. J Oral Maxillofac Surg. 2006;64(4):708-711. doi:10.1016/j.joms.2005.12.029

79. Kojima $M$, Itoh $H$, Hirabayashi $K$, et al. Methotrexate-associated lymphoproliferative disorders. A clinicopathological study of 13 Japanese cases. Pathol Res Pract. 2006;202(9):679-685. doi:10.1016/j.prp.2006.05.007

80. Muirhead R, Ritchie DM. Partial regression of Merkel cell carcinoma in response to withdrawal of azathioprine in an immunosuppressioninduced case of metastatic Merkel cell carcinoma. Clin Oncol (R Coll Radiol). 2007;19(1):96. doi:10.1016/j.clon.2006.10.001

81. Tanaka A, Shigematsu H, Kojima M, Sakashita H, Kusama K. Methotrexate-associated lymphoproliferative disorder arising in a patient with adult Still's disease. J Oral Maxillofac Surg. 2008;66(7):1492-1495. doi:10.1016/j.joms.2007.05.006

82. Uneda S, Sonoki T, Nakamura Y, Matsuoka H, Nakakuma H. Rapid vanishing of tumors by withdrawal of methotrexate in EpsteinBarr virus-related B cell lymphoproliferative disorder. Intern Med. 2008;47(15):1445-1446. doi:10.2169/internalmedicine.47.0989

83. Hasserjian RP, Chen S, Perkins SL, et al. Immunomodulator agent-related lymphoproliferative disorders. Mod Pathol. 2009;22(12):1532-1540. doi:10.1038/modpathol.2009.131

84. Dojcinov SD, Venkataraman G, Raffeld M, Pittaluga S, Jaffe ES. EBV positive mucocutaneous ulcer - a study of 26 cases associated with various sources of immunosuppression. Am J Surg Pathol. 2010;34(3):405-417. doi:10.1097/PAS.0b013e3181cf8622

85. Kikuchi K, Miyazaki Y, Tanaka A, et al. Methotrexate-related Epstein-Barr virus (EBV)-associated lymphoproliferative disorder - so-called "Hodgkin-like lesion" - of the oral cavity in a patient with rheumatoid arthritis. Head Neck Pathol. 2010;4(4):305-311. doi:10.1007/s12105-010-0202-6

86. Mattsson U, Magnusson B, Jontell M. Squamous cell carcinoma in a patient with oral lichen planus treated with topical application of tacrolimus. Oral Surg Oral Med Oral Pathol Oral Radiol Endod. 2010;110(1):e19-e25. doi:10.1016/j.tripleo.2010.02.030 
87. Güngör \$̧, Gökdemir G, Büyükbabani N, Bahçetepe N. Squamous cell carcinoma on the lower lip after using topical calcineurin inhibitor. J Dtsch Dermatol Ges. 2013;11(9):868-870. doi:10.1111/ddg.12133

88. Ishida M, Hodohara K, Yoshii $M$, et al. Methotrexate-related Epstein-Barr virus-associated lymphoproliferative disorder occurring in the gingiva of a patient with rheumatoid arthritis. Int J Clin Exp Pathol. 2013;6(10):2237-2241.

89. Horie N, Kawano R, Kaneko T, Shimoyama T. Methotrexate-related lymphoproliferative disorder arising in the gingiva of a patient with rheumatoid arthritis. Aust Dent J. 2015;60(3):408-411. doi:10.1111/adj.12235

90. Tokuyama R, Sato T, Tatehara S, et al. Methotrexate-associated lymphoproliferative disorder complicated by bisphosphonaterelated osteonecrosis of the jaw arising in a female rheumatoid arthritis patient: Report of a case. J Oral Maxillofac Surg Med Pathol. 2014;26(3):374-378. doi:10.1016/j.ajoms.2014.01.004

91. Hashimoto K, Nagao T, Saito T, Kinoshita H. Methotrexateassociated lymphoproliferative disorders of the tongue developing in patients with rheumatoid arthritis: A report of 2 cases and a review. OralSurg OralMed Oral Pathol Oral Radiol. 2015;119(1):e1-e5. doi:10.1016/j.0ooo.2014.04.002

92. Miyashita M, Koike T, Aizawa H, Kurita H. Reversible methotrexateassociated lymphoproliferative disorder (MTX-LPD) associated with Epstein-Barr virus in a patient with rheumatoid arthritis. J Oral Maxillofac Surg Med Pathol. 2015;27(5):673-676. doi:10.1016/j.ajoms.2014.11.012

93. Xu G, Wang B, Yang M, Qian W. A rare case of nasopharyngeal carcinoma in a patient with multiple myeloma after treatment by lenalidomide. Int J Clin Exp Pathol. 2015;8(11):15025-15029.

94. Chambon F, Osdoit S, Bagny K, Moro A, Nguyen J, Réguerre $Y$. Dramatic response to nivolumab in xeroderma pigmentosum skin tumor. Pediatr Blood Cancer. 2018;65(2). doi:10.1002/pbc.26837

95. Lavacchi D, Nobili S, Brugia M. A case report of eyelid Merkel cell carcinoma occurring under treatment with nivolumab for a lung adenocarcinoma. BMC Cancer. 2018;18:1024. doi:10.1186/s12885-018-4919-z 